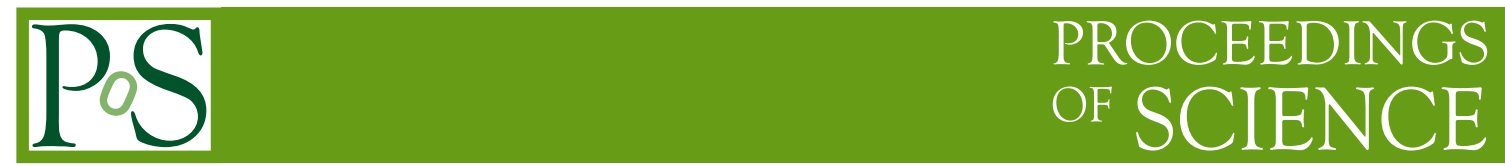

\title{
Search for Di-Higgs Production with the ATLAS Detector
}

\section{Tulin Varol*}

Southern Methodist University

On behalf of the ATLAS Collaboration

E-mail: tulin.varol@cern.ch

An enhanced production of two Higgs bosons would be a clear sign of beyond Standard Model physics. A search is performed for resonant and non-resonant excess production, including several decay channels of the two Higgs bosons. The analysis uses about $10 \mathrm{fb}^{-1}$ of $p p$ collisions at $\sqrt{s}=13 \mathrm{TeV}$.

38th International Conference on High Energy Physics 3-10 August 2016

Chicago, USA

${ }^{*}$ Speaker. 


\section{Introduction}

A particle consistent with the Standard Model (SM) Higgs boson $(h)$ was discovered in 2012 by both the ATLAS [1] and CMS [2] experiments at the Large Hadron Collider (LHC) [3, 4]. Further studies of its properties [5-8], such as the measurement of its spin and couplings, showed no significant deviations from the SM expectation. The discovery of the Higgs boson provided an important window on physics beyond the SM.

The predicted rate of the Higgs boson pair production in the SM is several orders of magnitude smaller than the rate for the single $h$ process $[9,10]$. It is not expected to be observable with current LHC data sets. However, many new physics models predict rates of Higgs boson pair production significantly higher than the SM rate. Enhancements of the expected SM production cross-section take two basic forms: non-resonant and resonant. For instance, any modification of the SM couplings, a deviation in the Higgs boson self-coupling $\lambda_{h h h}$, or additional tthh vertex (absent in SM) would lead to a non-resonant cross-section enhancement. The enhancement of the two Higgs production rate could be also due to the presence of $\mathrm{TeV}$ scale resonances such as the first Kaluza-Klein (KK) excitation of the spin-2 graviton $G_{K K}^{*}$ predicted by the bulk Randall-Sundrum (RS) model [11,12] or the heavy neutral scalar $H$ of two-Higgs-doublet models [13].

Searches for both non-resonant and resonant production of Higgs boson pairs in $b \bar{b} b \bar{b}$ [14], $\gamma \gamma W W^{*}[15]$ and $\gamma \gamma b \bar{b}$ [16] decay channels using Run-2 ATLAS data are presented in this document.

\section{Search for pair production of Higgs bosons in the $b \bar{b} b \bar{b}$ final state}

A search for the Higgs boson pair production in the $b \bar{b} b \bar{b}$ final state is carried out using 13.3 $\mathrm{fb}^{-1}$ of $p p$ collision data collected at $\sqrt{s}=13 \mathrm{TeV}$ in 2015 and 2016 [14]. This final state benefits from large $h \rightarrow b b$ branching fraction of 58\% [17]. Two methods, resolved and boosted, which are complementary in their acceptance, are used to reconstruct the Higgs boson. Both techniques use anti- $k_{t}$ jets [18] but with different jet radius parameter, $R$. The 'resolved' technique is optimised for the non-resonant or low mass $h h$ systems to enable the reconstruction of each $b$-quark as a distinct $b$-jet, whereas the 'boosted' technique is used to analyse higher mass resonant $h h$ systems in which two $b$-jets can not be resolved due to the higher Lorentz boost.

The data used in this analysis were recorded with a combination of multijet triggers which is more than $99.5 \%$ efficient for signal events satisfying the offline selection. The 2015 and 2016 datasets are analysed separately because LHC conditions such as instantaneous luminosity were different during these two years, necessitating the use of different trigger schemes. The results are then combined statistically.

In the analysis with the resolved method, candidate events are required to have at least four $b$-tagged anti- $k_{t} R=0.4$ jets with $p_{\mathrm{T}}>30 \mathrm{GeV}$ and $|\eta|<2.5$. A multivariate $b$-tagging algorithm $[19,20]$ with an estimated efficiency of $70 \%$ is used to tag jets containing $b$-hadrons. Four jets with the highest $b$-tagging score are selected. These are then used to form two Higgs boson candidates. Requirements on the angular distance between the jets $(\Delta R(j, j))$ that form each Higgs candidate are applied as a function of $m_{4 \mathrm{j}}$. In the absence of energy loss through semi-leptonic decays, the optimal choice of the jet pair would be the combination most consistent with the decays of two particles of equal mass. To account for energy loss, the choice is modified to the pairing that 
minimizes $D_{h h}$ of [14], defined in the $m_{2 \mathrm{j}}^{\text {lead }}-m_{2 \mathrm{j}}^{\text {subl }}$ plane as a distance from a line connecting ( 0 $\mathrm{GeV}, 0 \mathrm{GeV})$ and $(120 \mathrm{GeV}, 115 \mathrm{GeV})$. The values of $120 \mathrm{GeV}$ and $115 \mathrm{GeV}$ are the centre of the signal region, respectively, which correspond to the median values of the narrowest intervals that contain $90 \%$ of the signal in simulations.

Due to different kinematics for different resonance mass regions, the optimal selection in lowmass resonances differs from the optimal selection for higher masses. In order to enhance the analysis sensitivity, the requirements as a function of the reconstructed resonance mass $m_{4 \mathrm{j}}$ are applied on the three variables; leading and sub-leading Higgs boson candidate $p_{\mathrm{T}}$ values, and the pseudo-rapidity difference between the two Higgs boson candidates, $\left|\Delta \eta_{h h}\right|$. A further requirement is made on the angular separation in $\eta-\phi$ space between two Higgs boson candidates, $\Delta R_{h h}$, demanding to be greater than 1.5 .

After the full event selection, about $95 \%$ of the total background consists of multijet events, while the remaining $5 \%$ is mostly due to $t \bar{t}$ events. Background from all other sources is negligible. The $t \bar{t}$ contribution is taken from Monte Carlo (MC) simulation normalized to the data in the dedicated control samples. The multijet background is modelled using a fully data-driven approach in '2-tag' data sample passing the same selection as the signal except that exactly two jets must pass the $b$-tagging requirement. A signal-free sideband region is used to extrapolate 2-tag events to 4-tag signal region. The 2-tag sample is corrected for the kinematic differences arising from the additional $b$-tagging requirements in the signal sample. The modelling is verified in a control region, which is also used to assess systematic uncertainties. Both sideband and control regions are chosen to be orthogonal to the signal region, defined as:

$$
X_{h h}=\sqrt{\left(\frac{m_{2 \mathrm{j}}^{\text {lead }}-120 \mathrm{GeV}}{0.1 m_{2 \mathrm{j}}^{\text {lead }}}\right)^{2}+\left(\frac{m_{2 \mathrm{j}}^{\text {subl }}-115 \mathrm{GeV}}{0.1 m_{2 \mathrm{j}}^{\text {subl }}}\right)^{2}}<1.6 .
$$

The boosted analysis requires events with at least two anti- $k_{t} R=1.0$ (large- $R$ ) jets with $p_{\mathrm{T}}>250$ $\mathrm{GeV},|\eta|<2.0$ and mass $m_{J}>50 \mathrm{GeV}$. The two jets with highest $p_{\mathrm{T}}$ are selected. The leading jet is additionally required to have $p_{\mathrm{T}}>450 \mathrm{GeV}$ in order to suppress contamination from $t \bar{t}$ events. The two large- $R$ jets are required to have a separation $|\Delta \eta|<1.7$ to suppress contamination from multijet events. At least one $b$-tagged $R=0.2$ track jet, with $p_{\mathrm{T}}>10 \mathrm{GeV}$ and $|\eta|<2.5$, is required to be associated to each large- $R$ jet.

Depending on the total number of $b$-tagged track-jets associated to the Higgs boson candidates, three separate samples of events are defined. The 'two-tag-split' sample requires each Higgs boson candidate to have exactly one associated $b$-tagged track-jet. In the 'three-tag' and 'four-tag' samples, there are exactly three or exactly four b-tagged track-jets associated to Higgs boson candidates in the event, with two $b$-tagged jets associated with one candidate and one or two associated with the other candidate.

Analogously to the resolved analysis, the dominant multijet background is modelled by using data in the sideband region. The remaining $t \bar{t}$ background is also derived in this region using data while the shape is taken from the MC simulation. These background models are verified in the control region. The signal region definition is similar to the one used in the resolved analysis, shown by Eq. 2.1, but replacing $\left(m_{2 \mathrm{j}}^{\text {lead }}, m_{2 \mathrm{j}}^{\text {subl }}\right)$ by $\left(m_{\mathrm{J}}^{\text {lead }}, m_{\mathrm{J}}^{\text {subl }}\right)$. The non-resonant search is performed using only results from the resolved analysis, since it has better sensitivity than the boosted analysis. The 
observed 95\% CL upper limit is $\sigma(p p \rightarrow h h \rightarrow b \bar{b} b \bar{b})<330 \mathrm{fb}, 29$ times the SM predicted cross-section of $11.3_{-1.0}^{+0.9} \mathrm{fb}$.

For the resonant search, the results from the resolved and boosted analyses are used in complementary resonance mass regions where they offer their best sensitivity. The results of the resolved analysis are used up to a resonance mass of $1000 \mathrm{GeV}$ and those of the boosted analysis are used at higher mass where its expected sensitivity is higher. Fig. 1 shows the 95\% CL upper limits set for a spin-2 $G_{K K}^{*}$ in the bulk RS model with $k / \bar{M}_{P l}=1$ ( $k$ is the curvature of the warped extra dimension and $\bar{M}_{P l}=2.4 \times 10^{18} \mathrm{GeV}$ is the effective four-dimensional Planck scale). The resulting upper limit on $\sigma\left(p p \rightarrow G_{K K}^{*} \rightarrow h h \rightarrow b \bar{b} b \bar{b}\right)$ ranges from $1000 \mathrm{fb}$ at $m_{\mathrm{X}}=300 \mathrm{GeV}$ to $2 \mathrm{fb}$ at $m_{\mathrm{X}}=3000 \mathrm{GeV}$.

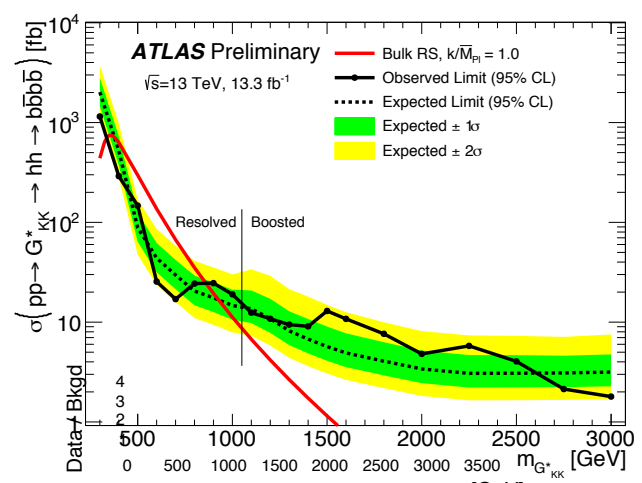

Figure 1: The expected and observed upper limit for $\sigma\left(p p \rightarrow G_{K K}^{*} \rightarrow h h \rightarrow b \bar{b} b \bar{b}\right)$ in the bulk RS model with $k / \bar{M}_{P l}=1$ at the $95 \% \mathrm{CL}$. The red curve shows the predicted cross-section as a function of resonance mass. The drop in cross-section for masses below $350 \mathrm{GeV}$ is due to a sharp drop in $\operatorname{BR}\left(G_{K K}^{*} \rightarrow h h\right)[14]$.

\section{Search for Higgs boson pair production in the final state of $\gamma \gamma W W^{*}(\rightarrow l v j j)$}

The resonant and non-resonant searches for Higgs boson pair production are performed using $13.3 \mathrm{fb}^{-1}$ of $p p$ collision data in the semi-leptonic $\gamma \gamma W W^{*}$ final state [15]. This analysis benefits from the large $h \rightarrow W W$ branching fraction and the excellent detector resolution for the diphoton mass. One of the $W$ bosons is required to decay to an electron or a muon (either directly or through a $\tau$ lepton) whereas the other is required to decay hadronically, resulting in the $\gamma \gamma l v q q^{\prime}$ final state.

Events are sampled using diphoton triggers that have approximately $100 \%$ efficiency for events passing the final offline selection. Events are required to have at least two photons, at least two jets and no $b$-jet in the final state. Leading (sub-leading) photon candidates are selected with $p_{\mathrm{T}} / m_{\gamma \gamma}$ $>0.35$ (0.25). Only events with $m_{\gamma \gamma}$ in the range of $105<m_{\gamma \gamma}<160 \mathrm{GeV}$ are considered. Two categories in the analysis are defined depending on the presence of lepton in the final state: a one-lepton region and a zero-lepton control region, by requiring at least one lepton or no lepton, respectively. The signal region is defined in the one-lepton region by requiring $m_{\gamma \gamma}$ to be within a $\pm 2 \sigma$ window of the Higgs boson mass $m_{h}$, where $m_{h}$ is of $125.09 \mathrm{GeV}$ and $\sigma_{\gamma \gamma}$ is of $1.7 \mathrm{GeV}$. Due to small number of events, both resonant and non-resonant searches proceed as counting experiments.

The main background sample consists of continuum background events with no Higgs boson. There is also a small contribution from single-Higgs processes to the total background. Similar to 
the $\gamma \gamma b \bar{b}$ analysis, single-Higgs processes are estimated from the MC simulation. The continuum background is modelled by using data in the sideband region defined by simply inverting the tight mass window requirement in one-lepton region. An unbinned fit to the $m_{\gamma \gamma}$ spectrum is performed to estimate the contribution from the continuum background events which is then extrapolated to the signal region. The left plot of the Fig. 2 shows the $m_{\gamma \gamma}$ distributions in the one-lepton region.

In the final signal region, there are 15 events observed in data and $7.88 \pm 1.24$ events expected from the backgrounds. The observed (expected) upper limit at 95\% CL on the cross-section of the non-resonant Higgs boson pair production is 25.0 (12.9) pb. The difference between expected and observed limits is expected since there is a slight excess of events in data. The observed (expected) upper limit on the cross-section times the branching fraction of $X \rightarrow h h$ ranges from 47.7 (24.3) pb to 24.7 (12.7) pb, as a function of $m_{\mathrm{X}}$ between 260 and $500 \mathrm{GeV}$, as shown in Fig. 2 (on the right).
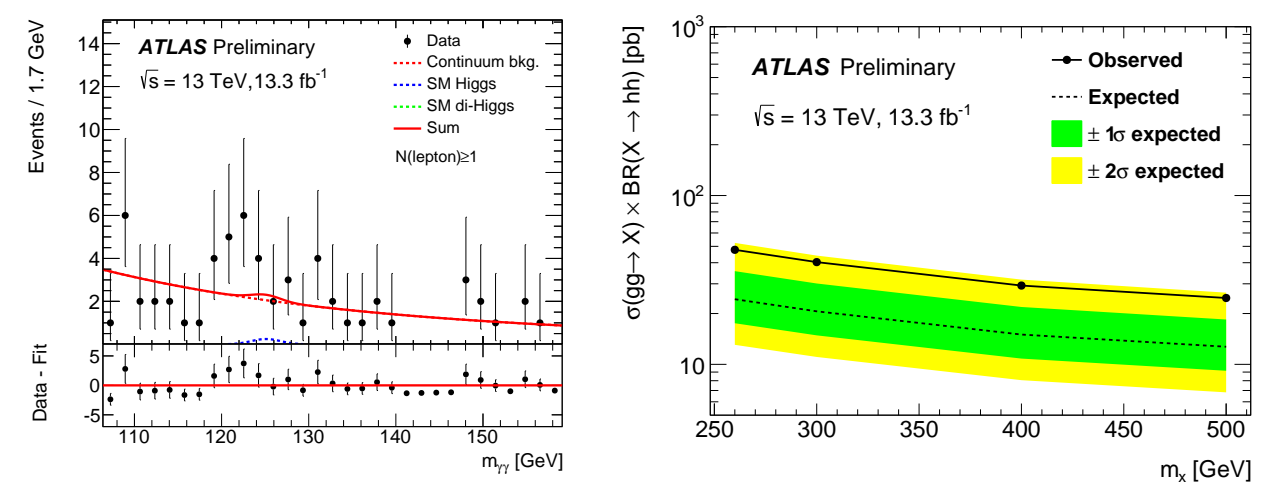

Figure 2: Left: Invariant mass spectrum of the diphoton system in the searches for both nonresonant and resonant Higgs boson pair production, with the corresponding estimations of the continuum and SM single-Higgs backgrounds for the one-lepton region. Right: 95\% CL expected (dashed line) and observed (solid line) limits on the resonant Higgs boson pair production. cross section times the branching fraction of $X \rightarrow h h$ as a function of $m_{\mathrm{X}}$ [15].

\section{Search for Higgs boson pair production in the $b b \gamma \gamma$ final state}

Searches for both resonant and non-resonant Higgs boson pair production are performed in the $\gamma \gamma b \bar{b}$ final state using $3.2 \mathrm{fb}^{-1}$ of $p p$ collision data recorded at $\sqrt{s}=13 \mathrm{TeV}$ in 2015 [16]. This final state benefits from large $h \rightarrow b b$ branching fraction, relatively small backgrounds and excellent diphoton mass resolution.

The data were recorded with diphoton triggers that are nearly $100 \%$ efficient for events satisfying the photon requirements. Events with at least two isolated photons and exactly two $b$-tagged jets are selected. The leading (sub-leading) photon $p_{\mathrm{T}}$ is required to be larger than $35 \%(25 \%)$ of the diphoton invariant mass $m_{\gamma \gamma}$, which itself is chosen to lie in the range of $105<m_{\gamma \gamma}<160$ $\mathrm{GeV}$. The leading (sub-leading) $b$-jet $p_{\mathrm{T}}$ is required to be greater than $55(35) \mathrm{GeV}$. The invariant mass of the $b$-jet pair $m_{b \bar{b}}$ must fall within a mass window of $95<m_{b \bar{b}}<135 \mathrm{GeV}$. The sample of events passing the selection criteria is defined as 2-tag events, while events with no $b$-tagged jets but passing all other requirements are called as 0-tag events. Events in 2-tag sample are used to test the presence of signal whereas 0 -tag events are used to model the continuum background. The other background process for this analysis consist of events containing a single Higgs boson. 
In the non-resonant search, an unbinned fit to the $m_{\gamma \gamma}$ distribution is performed simultaneously in the 0 -tag and 2-tag regions. The shapes of the $m_{\gamma \gamma}$ resonance for simulated di-Higgs and singleHiggs events are described by the double-sided Crystal Ball functions [21]. In order to describe the shape of the continuum background, the exponential fit is performed to $m_{\gamma \gamma}$ in the sidebands which is defined as the region $105 \mathrm{GeV}<m_{\gamma \gamma}<160 \mathrm{GeV}$ but excluding the window of $m_{h} \pm 2 \sigma_{m_{\gamma \gamma}}$. The slope of the exponential is shared in the fit between 0- and 2-tag categories so that the control region constrains the background shape in the signal region.

The resonant search proceeds as a counting experiment with requirements both in $m_{\gamma \gamma}$ and $m_{\gamma \gamma b \bar{b}}$. The $m_{\gamma \gamma b \bar{b}}$ signal window is defined to be twice the mass resolution $2 \sigma_{m_{\gamma \gamma b \bar{b}}}$ around the Higgs boson mass $m_{h}$. After $b b$ mass is constrained to $m_{h}$, an additional requirement on $m_{\gamma \gamma b \bar{b}}$ is defined as the smallest window containing $95 \%$ of the signal events based on MC simulation. As in the non-resonant search, the single-Higgs background is estimated from the MC simulation. The continuum background in the $m_{\gamma \gamma b \bar{b}}$ signal window is extrapolated from the diphoton mass sidebands.

In the non-resonant search, within the $m_{\gamma \gamma b \bar{b}}$ signal window, 1.8 events are expected from the combination of continuum and single-Higgs backgrounds while none are observed. Assuming SM branching fractions, a 95\% CL upper limit of 3.9 (5.4) pb is observed (expected) for $\sigma(g g \rightarrow h h)$. For the resonant searches, the observed (expected) upper limits on $\sigma(g g \rightarrow H \rightarrow h h)$ at $95 \% \mathrm{CL}$ vary from $7.0(7.5) \mathrm{pb}$ at $m_{H}=275 \mathrm{GeV}$ to $4.0(4.4) \mathrm{pb}$ at $m_{H}=400 \mathrm{GeV}$ for a spin-zero resonant state in the narrow-width approximation.
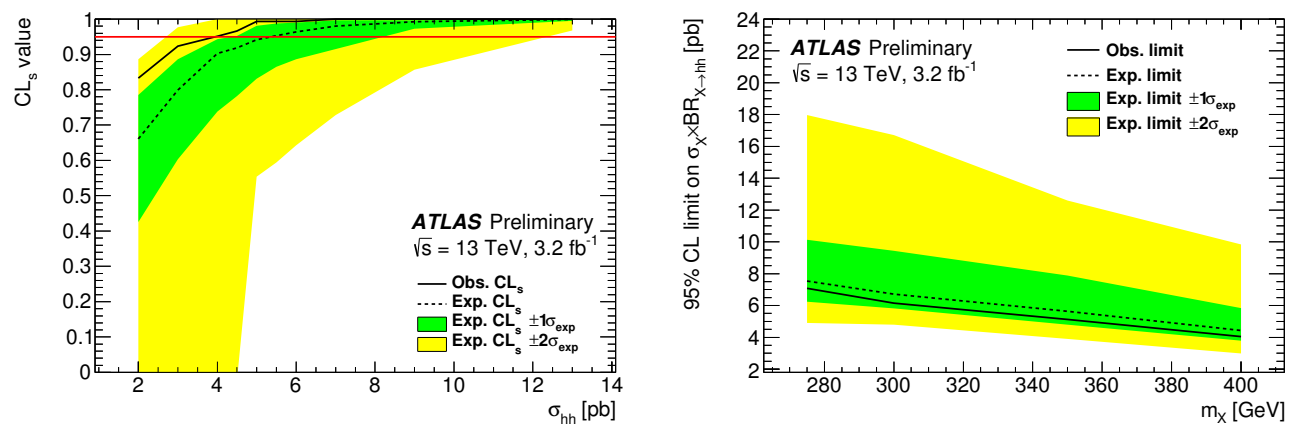

Figure 3: Left: Scan of the observed (solid line) and expected (dashed line) $\mathrm{CL}_{S}$ values as a function of the production cross-section $\sigma_{h h}$ in the search for the non-resonant di-Higgs production. The green (yellow) band represents the $1 \sigma(2 \sigma)$ intervals on the expected $\mathrm{CL}_{\mathrm{S}}$ value. Right: $95 \%$ CL expected (dashed line) and observed (solid line) limits on the production cross-section times $X \rightarrow h h$ branching fraction in the resonant search [16].

\section{Conclusion}

Most recent searches for Higgs boson pair production using data collected with the ATLAS detector are highlighted. Results for $b \bar{b} b \bar{b}$ and $W W y y$ analyses are based on the analysis of data collected at $\sqrt{s}=13 \mathrm{TeV}$ corresponding to an integrated luminosity of $13.3 \mathrm{fb}^{-1}$. The $\gamma \gamma b \bar{b}$ analysis is performed only with data collected in 2015 corresponding to an integrated luminosity of $3.2 \mathrm{fb}^{-1}$. No significant excess beyond the Standard Model expectations is observed in any of the analysis. Hence, limits are set on various observables. 


\section{References}

[1] ATLAS Collaboration, G. Aad et al., The ATLAS Experiment at the CERN Large Hadron Collider, JINST 3 (2008) S08003.

[2] CMS Collaboration, S. Chatrchyan et al., The CMS experiment at the CERN LHC, JINST 3 (2008) S08004.

[3] ATLAS Collaboration, G. Aad et al., Observation of a new particle in the search for the Standard Model Higgs boson with the ATLAS detector at the LHC, Phys. Lett. B716 (2012) 1-29, arXiv:1207.7214 [hep-ex].

[4] CMS Collaboration, S. Chatrchyan et al., Observation of a new boson at a mass of $125 \mathrm{GeV}$ with the CMS experiment at the LHC, Phys. Lett. B716 (2012) 30-61, arXiv:1207. 7235 [hep-ex] .

[5] ATLAS Collaboration, G. Aad et al., Study of the spin and parity of the Higgs boson in diboson decays with the ATLAS detector, Eur. Phys. J. C75 no. 10, (2015) 476, arXiv: 1506.05669 [hep-ex ], [Erratum: Eur. Phys. J.C76,no.3,152(2016)].

[6] ATLAS Collaboration, G. Aad et al., Measurements of the Higgs boson production and decay rates and coupling strengths using pp collision data at $\sqrt{s}=7$ and $8 \mathrm{TeV}$ in the ATLAS experiment, Eur. Phys. J. C76 no. 1, (2016) 6, arXiv: 1507.04548 [hep-ex] .

[7] CMS Collaboration, V. Khachatryan et al., Precise determination of the mass of the Higgs boson and tests of compatibility of its couplings with the standard model predictions using proton collisions at 7 and 8 TeV, Eur. Phys. J. C75 no. 5, (2015) 212, arXiv:1412.8662 [hep-ex] .

[8] V. Khachatryan et al., Limits on the Higgs boson lifetime and width from its decay to four charged leptons, Phys. Rev. D92 no. 7, (2015) 072010, arXiv:1507.06656 [hep-ex] .

[9] The LHC Higgs Cross Section Working Group, Current recommendations for di-Higgs cross-sections, https://twiki.cern.ch/twiki/bin/view/LHCPhysics/ LHCHXSWGHH\#Current_recommendations_for_di_H, 2016.

[10] LHC Higgs Cross Section Working Group Collaboration, D. de Florian et al., Handbook of LHC Higgs Cross Sections: 4. Deciphering the Nature of the Higgs Sector, arXiv: 1610.07922 [hep-ph].

[11] A. L. Fitzpatrick, J. Kaplan, L. Randall, and L.-T. Wang, Searching for the Kaluza-Klein Graviton in Bulk RS Models, JHEP 09 (2007) 013, arXiv: hep-ph/ 0701150 [hep-ph ] .

[12] K. Agashe, H. Davoudiasl, G. Perez, and A. Soni, Warped Gravitons at the LHC and Beyond, Phys. Rev. D76 (2007) 036006, arXiv : hep-ph/ 0701186 [hep-ph] .

[13] G. C. Branco, P. M. Ferreira, L. Lavoura, M. N. Rebelo, M. Sher, and J. P. Silva, Theory and phenomenology of two-Higgs-doublet models, Phys. Rept. 516 (2012) 1-102, arXiv: 1106.0034 [hep-ph].

[14] ATLAS Collaboration, Search for pair production of Higgs bosons in the $b \bar{b} b \bar{b}$ final state using proton-proton collisions at $\sqrt{s}=13 \mathrm{TeV}$ with the ATLAS detector, Tech. Rep.

ATLAS-CONF-2016-049, CERN, Geneva, Aug, 2016.

https: //cds.cern. ch/record/2206131.

[15] ATLAS Collaboration, Search for Higgs boson pair production in the final state of $\gamma \gamma W W^{*}(\rightarrow l v j j)$ using $13.3 \mathrm{fb}^{-1}$ of pp collision data recorded at $\sqrt{\mathrm{s}}=13 \mathrm{TeV}$ with the ATLAS detector, Tech. Rep. ATLAS-CONF-2016-071, CERN, Geneva, Aug, 2016. https://cds.cern. ch/record/2206222. 


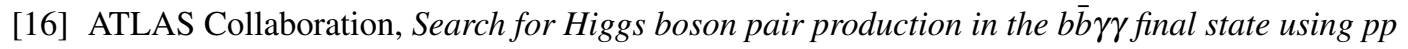
collision data at $\sqrt{s}=13 \mathrm{TeV}$ with the ATLAS detector, Tech. Rep. ATLAS-CONF-2016-004, CERN, Geneva, Mar, 2016. https : / / cds. cern. ch/record/2138949.

[17] The LHC Higgs Cross Section Working Group, SM Higgs Branching Ratios and Total Decay Widths, https:

//twiki.cern.ch/twiki/bin/view/LHCPhysics/CERNYellowReportPageBR, 2016.

[18] M. Cacciari, G. P. Salam, and G. Soyez, The Anti-k(t) jet clustering algorithm, JHEP 04 (2008) 063, arXiv:0802.1189 [hep-ph].

[19] ATLAS Collaboration, Optimisation of the ATLAS b-tagging performance for the 2016 LHC Run, Tech. Rep. ATL-PHYS-PUB-2016-012, CERN, Geneva, Jun, 2016.

https://cds.cern.ch/record/2160731.

[20] ATLAS Collaboration, G. Aad et al., Performance of b-Jet Identification in the ATLAS Experiment, JINST 11 no. 04, (2016) P04008, arXiv: 1512.01094 [hep-ex] .

[21] ATLAS Collaboration, G. Aad et al., Search for Scalar Diphoton Resonances in the Mass Range $65-600 \mathrm{GeV}$ with the ATLAS Detector in pp Collision Data at $\sqrt{s}=8 \mathrm{TeV}$, Phys. Rev. Lett. 113 no. 17, (2014) 171801, arXiv:1407.6583 [hep-ex] . 